\title{
MICOPLASMOSE EM FELINO DOMÉSTICO, FELV (+), RELATO DE CASO
}

\author{
Alexsander Ferraz ${ }^{1}$ \\ Eugênia Tavares Barwaldt ${ }^{2}$ \\ Bruna dos Santos Pires ${ }^{3}$ \\ Camila Moura de Lima ${ }^{4}$ \\ Eduarda Santos Bierhals ${ }^{5}$ \\ Márcia de Oliveira Nobre ${ }^{6}$ \\ Leandro Quintana Nizoli ${ }^{7}$
}

\begin{abstract}
RESUMO
Mycoplasma spp. são bactérias pleomórficas que parasitam a superfície das hemácias de várias espécies domésticas, sendo o Mycoplasma haemofelis, a espécie mais frequente nos felinos. Este parasito é reponsável pela micoplasmose felina, também chamada de micoplasmose haemotrópica felina (MHF) e de anemia infecciosa felina, que pode causar um quadro de anemia hemolítica aguda ou crônica. O objetivo deste trabalho, foi relatar o caso de um felino doméstico, apresentando na avaliação clínica, mucosas ictéricas e os linfonodos submandibulares aumentados de volume. Foi realizado teste rápido para diagnóstico de FIV/FeLV, onde constatou-se que o paciente era FeLV (+), sendo este, um fator de risco para o desenvolvimento da micoplasmose. No eritrograma, evidenciou-se diminuição do hematócrito, hemácias e hemoglobina. O exame bioquímico, indicou aumento de ALT, ureia e bilirrubina total. Para diagnóstico definitivo, foi realizado pesquisa de hemoparasitos, pela técnica de esfregaço sanguíneo corado, onde observou-se na superfície das hemácias, a presença de estruturas eosinofílicas na forma de cocos, característicos do gênero Mycoplasma.
\end{abstract}

Palavras chave: hemoplasma, gato doméstico, diagnóstico.

\section{MYCOPLASMOSIS IN FELINE DOMESTIC, FeLV (+), CASE REPORT}

\begin{abstract}
Mycoplasma spp. they are pleomorphic bacteria that parasitize the surface of red blood cells of several domestic species, Mycoplasma haemofelis being the most frequent species in cats. This parasite is responsible for feline mycoplasmosis, also called feline haemotropic mycoplasmosis (MHF) and feline infectious anemia, which can cause acute or chronic hemolytic anemia. The aim of this study was to report the case of a domestic feline, presenting in the clinical evaluation, jaundiced mucous membranes and enlarged submandibular lymph nodes. A rapid test was performed for the diagnosis of FIV / FeLV, where it was found that the patient was FeLV (+),

\footnotetext{
${ }^{1}$ Doutorando do Programa de Pós Graduação em Veterinária da Universidade Federal de Pelotas. Correspondência: xanderferraz@yahoo.com.br.

${ }^{2}$ Graduando em medicina Veterinária Universidade Federal de Pelotas. tbeugenia@gmail.com.

${ }^{3}$ Residente em Clínica Cirúrgica de Animais de Companhia Universidade Federal de Pelotas, bruspires@gmail.com.

${ }^{4}$ Mestranda do Programa de Pós Graduação em Veterinária da Universidade Federal de Pelotas. camila.moura.lima@hotmail.com.

${ }^{5}$ Residente em Clínica Médica de Animais de Companhia na Universidade Federal de Pelotas. dudabierhals@hotmail.com.

${ }^{6}$ Professor Associado da Universidade Federal de Pelotas. marciaonobre@gmail.com.

${ }^{7}$ Professor Adjunto da Universidade Federal de Pelotas. leandro.nizoli@gmail.com.
} 
which is a risk factor for the development of mycoplasmosis. The erythrogram showed a decrease in hematocrit, red blood cells and hemoglobin. Biochemical examination indicated an increase in ALT, urea and total bilirubin. For a definitive diagnosis, a survey of hemoparasites was carried out, using the stained blood smear technique, where the presence of eosinophilic

structures in the form of coconuts, characteristic of the genus Mycoplasma, was observed on the surface of red blood cells.

Keywords: hemoplasma, domestic cat, diagnosis.

\section{MICOPLASMOSIS EN FELINO DOMÉSTICO, FeLV (+), CASO CLÍNICO}

\section{RESUMEN}

Mycoplasma spp. son bacterias pleomórficas que parasitan la superficie de los glóbulos rojos de varias especies domésticas, siendo Mycoplasma haemofelis la especie más frecuente en gatos. Este parásito es responsable de la micoplasmosis felina, también llamada micoplasmosis hemotrópica felina (MHF) y anemia infecciosa felina, que puede causar anemia hemolítica aguda o crónica. El objetivo de este trabajo fue informar el caso de un felino doméstico, presentando en la evaluación clínica, membranas mucosas con ictericia y ganglios linfáticos submandibulares agrandados. Se realizó una prueba rápida para el diagnóstico de FIV / FeLV, donde se encontró que el paciente era FeLV (+), que es un factor de riesgo para el desarrollo de micoplasmosis. El eritrograma mostró una disminución en el hematocrito, los glóbulos rojos y la hemoglobina. Para un diagnóstico definitivo, se realizó una encuesta de hemoparásitos, utilizando la técnica de frotis de sangre teñida, donde se observó la presencia de estructuras eosinofílicas en forma de cocos, características del género Mycoplasma, en la superficie de los glóbulos rojos.

Palabras clave: hemoplasma, gato doméstico, diagnóstico.

\section{INTRODUÇÃO}

A micoplasmose felina, também chamada de micoplasmose haemotrópica felina (MHF) ou até mesmo de anemia infecciosa felina, é causada por parasitos do gênero Mycoplasma, sendo o Mycoplasma haemofelis, o mais frequente, candidatus Mycoplasma haemominutum, $e$ candidatus Mycoplasma turicensis também podem infectar os felinos (1). São bactérias pleomórficas de forma cocóide, gram-negativa, intracelulares obrigatórias e epi eritrocitárias (2).

Essa patologia na maioria dos casos relatados é de caráter subclínico, entretanto, a adesão deste parasito à superfície dos eritrócitos, pode levar à sua destruição pelo sistema fagocítico mononuclear, ocasionando um quadro de anemia hemolítica aguda ou crônica, de caráter leve a grave $(3,4)$.

A maioria dos gatos parasitados, encontram-se assintomáticos, mas quando presentes, os sinais clínicos incluem, anorexia, febre, perda de peso e mucosas pálidas (5), esplenomegalia, icterícia e linfadenopatia também podem estar presentes (6). 
É uma doença considerada de caráter oportunista, animais imunocompetentes geralmente não desenvolvem a sintomatologia da doença, sendo necessária uma imunossupressão para os sinais clínicos tornarem-se evidentes (7). Dentre os fatores de risco para desenvolvimento da doença, destacam-se, sexo, idade, acesso à rua, infecção concomitante com doenças imunossupressoras, como FIV e FELV (8), já que a FELV é dita como cofator para o desenvolvimento desse quadro (9). De acordo com Rand (2006), em zonas onde este vírus é comum, cerca de 50\% dos gatos está coinfectado com Mycoplasma haemofelis.

A principal forma de transmissão ocorre por meio de artrópodes, como pulgas (Ctenocephalides felis) e carrapatos (Rhipicephalus sanguineus), mas pode ocorrer por meio de brigas e mordeduras, onde haja o contato com sangue infectado, e de forma iatrogênica pela transfusão de sangue (8).

Este parasito assume importância na rotina clínica de pequenos animais e na saúde pública, pois também pode infectar o ser humano (10). O potencial zoonótico é evidenciado após identificação molecular de hemoplasmas em humanos e profissionais imunossuprimidos, alguns em contato frequente com animais infectados por Mycoplasma spp. $(11,12)$

\section{RELATO DE CASO}

Foi atendido no Hospital de Clinicas Veterinária (HCV) da UFPel, um felino, macho, de um ano e sem raça definida. No exame clinico geral, não apresentou alteração na temperatura retal $\left(38,6^{\circ} \mathrm{C}\right)$, frequência cardíaca $(160 \mathrm{bpm})$ e TPC $(<2$ ”). As mucosas encontravam-se levemente ictéricas e na avaliação dos linfonodos, os submandibulares apresentavam aumento de volume. Foi observado também, prurido na região do pescoço e cabeça, com alopecia e rarefação pilosa nas mesmas regiões. Durante a inspeção, foi observado presença de pulgas.

Foi realizado teste rápido para diagnóstico de FIV/FeLV, onde constatou-se que o paciente era FeLV (+). Na anamnese, os tutores foram questionados sobre cronograma de vacinação e controle de endo e ectoparasitas, onde os mesmos informaram que só realizavam a vermifugação, sendo que esta estava atrasada.

Pelo quadro clinico apresentado, juntamente com o diagnóstico de FeLV e a presença de pulgas, houve suspeita de micoplasmose felina. Uma amostra de sangue foi coletada para hemograma completo e análise bioquimica e também para pesquisa de hemoparasitos, através da técnica de esfregaço sanguíneo corado.

\section{DISCUSSÃO}

No eritrograma, observou-se diminuição de hematócrito (18\%), hemácias $\left(2,6 \times 10^{6} / \mu \mathrm{L}\right)$ e hemoglobina $(5,5 \mathrm{~g} / \mathrm{dL})$, e aumento do $\mathrm{VCM}(67,7 / \mathrm{fL})$, no diferencial, observou-se presença de metarrubrícitos (5\%), anisocitose e policromasia, e presença de corpúsculo de Howell-Jolly. Também foi observado, trombocitopenia $(141.000 / \mu \mathrm{L})$ e plasma ictérico. O M. haemofelis possui como característica aderir-se nos glóbulos vermelhos, desta forma, causando lise dos eritrócitos e proporcionando um quadro clínico de anemia leve a grave e icterícia. O grau de anemia está associado a intensidade da infecção e também ao sistema imunológico do paciente $(13,14)$. As alterações laboratoriais caracterizam-se por anemia regenerativa com anisocitose, policromasia, reticulocitose e corpúsculos de Howell-Jolly $(2,15)$, assim como foi observado neste caso. 
O exame bioquímico revelou aumento dos níveis de ALT (398 UI/L), em resposta a necrose hepática, secundária a anemia aguda; ureia $(80 \mathrm{mg} / \mathrm{dL})$, devido a ação nefrotóxica da hemoglobina circulante e bilirrubina total $(4,23 \mathrm{mg} / \mathrm{dL})$, devido a hemólise (16).

A literatura relata que felinos infectados pelo vírus da leucemia felina (FeLV) e/ou vírus da imunodeficiência felina (FIV) são mais propensos a serem infectados por Mycoplasma spp. Essa hipótese pode ser atribuída ao estilo de vida desses animais, onde a maioria vive ou tem acesso à rua, tornando-os mais suscetíveis as doenças retrovirais e a presença de ectoparasitas (17). Desta forma, pode-se realizar essa associação neste caso, pois o paciente mostrou-se positivo para FeLV, possuía acesso à rua e também tinha presença de pulgas.

No esfregaço sanguíneo, foi observado na superfície das hemácias, a presença de estruturas eosinofílicas na forma de cocos, característicos de Mycoplasma spp. (Figura 1). O diagnóstico pode ser obtido por meio de exames hematológicos, visualização do hemoparasito em esfregaço sanguíneo corado e também pelo teste da reação em cadeia da polimerase (PCR), que possui alta sensibilidade e permite a diferenciação entre as espécies de Mycoplasma $(13,16,15)$.

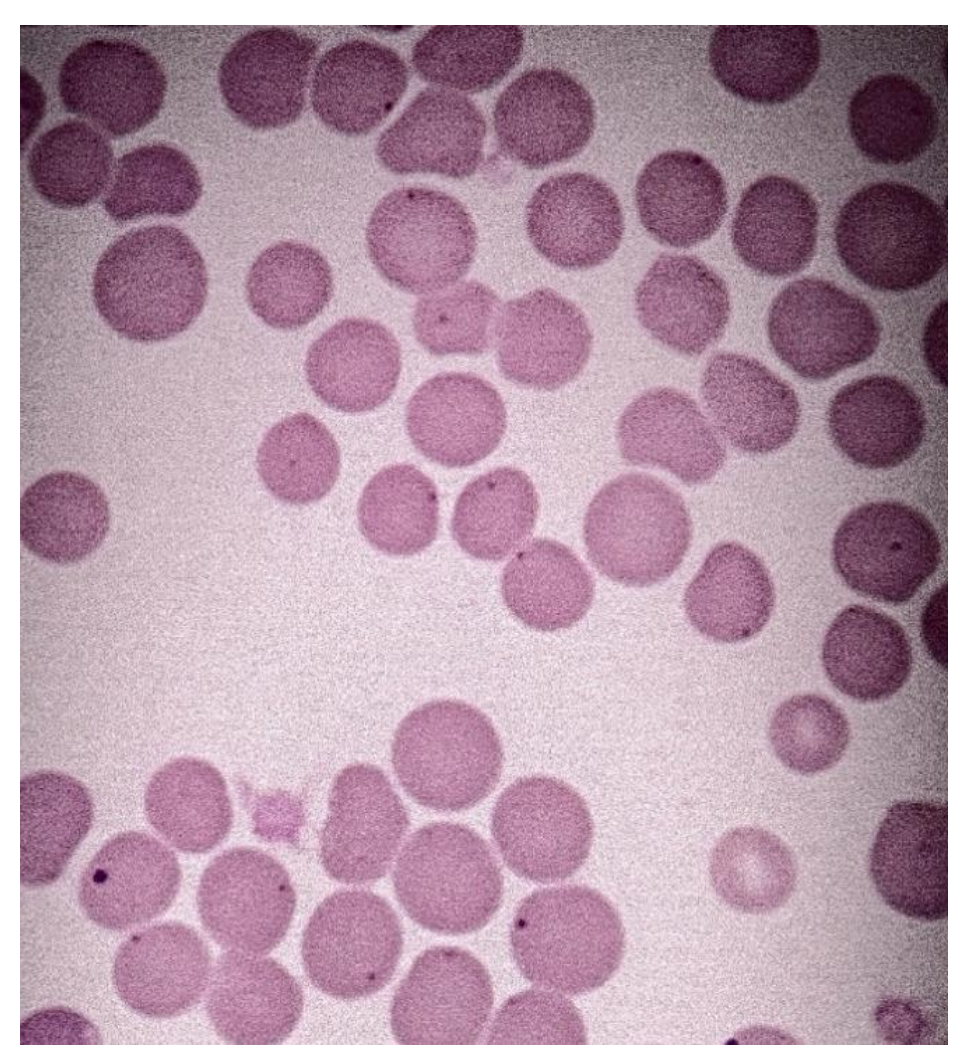

Figura 1. Mycoplasma spp. na superfície de hemácias, observados em microscopia ótica, aumento de $1000 \mathrm{X}$

O tratamento preconizado foi Doxiciclina, na dose de $5 \mathrm{mg} / \mathrm{kg}$, a cada 12 horas, durante 21 dias e Ácido Ursodesoxicólico, $15 \mathrm{mg} / \mathrm{kg}$, a cada 24 horas. Foi prescrito também, prednisolona, $0,5 \mathrm{mg} / \mathrm{kg}$, a cada 12 horas, timomodulina xarope, a cada 12 horas, durante 30 dias e o uso de antipulgas para o controle de ectoparasitos.

A doxiciclina é o antibiótico de eleição, por não causar nefrotoxicidade e ter baixa hepatotoxicidade nos gatos, comparado com outras tetraciclinas (2). Em animais com intensa anemia imuno-mediada, a prednisolona pode ser utilizada para ajudar a reduzir a eritrofagocitose, porém, apenas em casos onde o tratamento com antibiótico isolado não 
obtenha resposta satisfatória (13). Entretato, Willi et al. (18), não recomendam o uso dos corticosteroides, pois o efeito imunossupressor destes medicamentos, podem aumentar a parasitemia dos gatos infectados.

A timomodulina é utilizada com o intuito de proporcionar um aumento na resposta imunogência do paciente (19). Também deve ser realizado o controle de ectoparasitos, visto que a picada destes agentes é a principal forma de trasmissão. Nos casos de pacientes com anemia grave, é necessário tratamento de suporte com fluidoterapia e/ou transfusões sanguíneas $(20,21)$.

\section{CONCLUSÃO}

Este relato, evidencia a importância da pesquisa de hemoparasitos no diagnóstico de casos suspeitos de hemoparasitoses, como a Micoplasmose felina. Sendo o esfregaço sanguíneo uma das técnicas comumente utilizadas. A confirmação pelo exame laboratorial é de suma importância para que o tratamento ideal seja preconizado.

\section{REFERÊNCIAS}

1. Messick JB. Have the hemoplasmas evolved toward a "benign" coexistence with their host? In: Proceedings of 63rd Annual Meeting of the American College of Veterinary Pathologists; 2012; Seattle, Washington, USA. Seattle: ACVP; 2012.

2. Tasker S. Haemotropic mycoplasmas: what's their real significance in cats? J Feline Med Surg [Internet]. 2010 [cited 2019 Sept 15];12(5):369-81. Available from: https://reader.elsevier.com/reader/sd/pii/S1098612X1000104X?token=A2E717497F3F4B EC33EB2F0A967D12578C9E4B60986E8B4956528F0B01AF4C4E06AC677811C1A26B D5106B4CD1F49BE8

3. Neimark H, Johansson KE, Rikihisa Y, Tully JG. Proposal to transfer some members of the genera Haemobartonellaand Eperythrozoonto the genus Mycoplasmawith descriptions of Candidatus Mycoplasma haemofelis, Candidatus Mycoplasma haemomuris, Candidatus Mycoplasma haemosusisand, Candidatus Mycoplasma wenyonii. Int J Syst Evol Microbiol [Internet]. 2001 [cited 2020 Jun 24];51(Pt 3):891-9. Available from:

https://www.microbiologyresearch.org/docserver/fulltext/ijsem/51/3/0510891a.pdf?expires $=1595621783 \& \mathrm{id}=\mathrm{id} \&$ accname $=$ guest $\&$ checksum $=51$ B97432DBC5E3908D6EE5888F748 $\underline{\mathrm{A} 05}$

4. Messick JB. Hemotropic mycoplasmas (hemoplasmas): a review and new insights into pathogenic potential. Vet Clin Pathol. 2004;33(1):2-13.

5. Hicks CAE, Willi B, Riond B, Novacco M, Meli ML, Stokes CR, et al. Protective Immunity against Infection with Mycoplasma haemofelis. Clin Vaccine Immunol [Internet]. 2015

[cited 2020 Apr 10];22(2):108-18. Available from:

https://www.ncbi.nlm.nih.gov/pmc/articles/PMC4278926/pdf/zcd108.pdf 
6. Thrall MA, Weiser G, Allison RW, Campbell TW, editors. Veterinary hematology and clinical chemistry. 2nd ed. Ames: Wiley-Blackwell; 2012.

7. Costa HX. Interação de hemoparasitos e hemoparasitoses em casos clínicos de trombocitopenia em cães no município de Goiânia [dissertação]. Goiânia: Escola de Veterinária, Universidade Federal de Goiás; 2011.

8. Harvey JW. Hemotropic Mycoplasmosis (Hemobartonellosis). In: Greene CE, editor. Infectious diseases of dog and cat. 3rd ed. St. Louis: Saunders Elsevier; 2006. p.252-65.

9. Ramsey IK, Tennant BJ. BSAVA-Manual de doenças infeciosas em cães e gatos. 2a ed. São Paulo: Roca; 2010. Sistema linfopoético e linforeticular; p.87-70.

10. Duarte A, Marques V, Correia JHD, Neto I, São Bráz B, Rodrigues C, et al. Molecular detection of haemotropic Mycoplasma species in urban and rural cats from Portugal. $\mathrm{J}$ Feline Med Surg [Internet]. 2016 [cited 2020 Jun 24];17(6):516-22. Available from: https://journals.sagepub.com/doi/pdf/10.1177/1098612X14550172

11. Santos AP, Santos RP, Biondo AW, Dora JM, Goldani LZ, Oliveira ST, et al. Hemoplasma infection in HIV-positive patient, Brazil. Emerg Infect Dis [Internet]. 2008 [cited 2019

Sept 15];14(12):1922-4. Available from: https://www.ncbi.nlm.nih.gov/pmc/articles/PMC2634649/pdf/08-0964_finalD.pdf

12. Steer JA, Tasker S, Barker EN, Jensen J, Mitchell J, Stocki T, et al. A novel hemotropic Mycoplasma (hemoplasma) in a patient with hemolytic anemia and pyrexia. Clin Infect Dis [Internet]. 2011 [cited 2019 Sept 15];53(11):147-51. Available from: https://www.ncbi.nlm.nih.gov/pmc/articles/PMC3205199/pdf/cir666.pdf

13. Sykes JE. Feline hemotropic mycoplasmas. J Vet Emerg Crit Care [Internet]. 2010 [cited 2020 Apr 10];20(1):62-9. Available from: https://onlinelibrary.wiley.com/doi/abs/10.1111/j.1476-4431.2009.00491.x

14. Molina VM, Pacheco C. Manejo terapéutico de lipidosis hepática felina por Mycoplasma haemofelis en Medellín, Colombia: caso clínico. Rev Cientif [Internet]. 2016 [cited 2020 Jun 24];26(3):142-9. Available from: https://www.redalyc.org/pdf/959/95946430004.pdf

15. Raimundo JM, Guimarães A, Rodrigues RB, Botelho CFM, Peixoto MP, Pires MS, et al. Hematological changes associated with hemoplasma infection in cats in Rio de Janeiro, Brazil. Braz J Vet Parasitol [Internet]. 2016 [cited 2020 Apr 10];25(4):441-9. Available from: https://www.scielo.br/pdf/rbpv/v25n4/1984-2961-rbpv-S1984-29612016086.pdf

16. Martinez MS, Santos IFC, Kolber M, Poente MDP. Análise hematológica em gatos domésticos (Felis silvestris catus) diagnosticados com micoplasmose em Osasco, São Paulo - Brasil. Rev Lusófona Cienc Med Vet [Internet]. 2016 [cited 2020 Jun 24];8:1-9. Available from: https://revistas.ulusofona.pt/index.php/rlcmv/article/view/5702 
17. Bergmann M, Englert T, Stuetzer B, Hawley JR, Lappin MR, Hartmann K. Risk factors of different hemoplasma species infections in cats. BMC Vet Res [Internet]. 2017 [cited 2020 Jun 24];13:52. Available from: https://www.ncbi.nlm.nih.gov/pmc/articles/PMC5312425/pdf/12917_2017_Article_953.p $\underline{\mathrm{df}}$

18. Willi B, Boretti FS, Cattori V, Tasker S, Meli ML, Reusch C, et al. Identification, molecular characterization and experimental transmission of a new hemoplasma isolate from a cat with hemolytic anemia in Switzerland. J Clin Microbiol [Internet]. 2005 [cited 2020 Jun 24];43(6):2581-5. Available from: https://www.ncbi.nlm.nih.gov/pmc/articles/PMC1151947/pdf/2154-04.pdf

19. Almeida TM, Filho RPS, Cruz RO, Rodrigues APR, Silva ING. Linfoma leucemizado em felino coinfectado com o vírus da imunodeficiência felina e da leucemia felina: relato de caso. Arq Bras Med Vet Zootec [Internet]. 2019 [cited 2020 Apr 10];71(1):219-24.

Available from: https://www.scielo.br/pdf/abmvz/v71n1/0102-0935-abmvz-710100219.pdf

20. Silveira E, Pimentel MC, Marques SMT. Mycoplasma haemofelis em gato- relato de caso. Pubvet [Internet]. 2014 [cited 2019 Sept 15];8(13):1-7. Available from: https://www.pubvet.com.br/uploads/2cad0a9574db1df787bd2aa8cf12c496.pdf

21. Novacco M, Sugiarto S, Willi B, Baumann J, Spiri AM, Oestmann A, et al. Consecutive antibiotic treatment with doxycycline and marbofloxacin clears bacteremia in Mycoplasma haemofelis-infected cats. Vet Microbiol [Internet]. 2018 [cited 2020 Apr 10];217:112-20. Available from: https://www.sciencedirect.com/science/article/pii/S0378113517311689 